\title{
Correlations for Nusselt Number in Free Convection from an Isothermal Inclined Square Plate by a Numerical Simulation
}

\author{
Jasim Abdulateef ${ }^{1}$, Ayad Hassan ${ }^{2}$ \\ ${ }^{1}$ Mechanical Engineering Department, Diyala University, Diyala, Iraq \\ ${ }^{2}$ Material Engineering Department, University of Technology, Baghdad, Iraq \\ Email address: \\ jmabdulateef@gmail.com (J. Abdulateef), ayad_kadhm@yahoo.com (A. Hassan)
}

\section{To cite this article:}

Jasim Abdulateef, Ayad Hassan. Correlations for Nusselt Number in Free Convection from an Isothermal Inclined Square Plate by a Numerical Simulation. American Journal of Mechanics and Applications. Vol. 3, No. 2, 2015, pp. 8-18. doi: 10.11648/j.ajma.20150302.11

\begin{abstract}
This paper presents a numerical study on three-dimensional transient natural convection from an inclined isothermal square plate. The finite difference approach is used to solve the governing equations, in which buoyancy is modeled via the Boussinesq approximation. The complete Navier-Stokes equations are transformed and expressed in term of vorticity and vector potential. The transformed equations are solved using alternating direction implicit (ADI) method for parabolic portion of the problem and successive over relaxation (SOR) for the elliptic portion. Solutions for laminar case are obtained up to Grashof number of $5 \times 10^{4}$ as well as the inclination angles were varied from $0^{\circ}$ to $180^{\circ}$ with $30^{\circ}$ intervals, and the Prandtl number of 0.7 is considered. The results are shown in terms of isothermal plots, and the local and average Nusselt numbers are also presented. The simulation results show that the main process of heat transfer is conduction for Grashof number less than $10^{3}$ and convection for Grashof number larger than $10^{3}$. It is also found that, the values of Nusselt number show fairly large dependence on inclination angle and there is a significant difference in heat transfer rates between the upward and downward orientation. The average Nusselt number increases to $20 \%$ at the vertical position compared to horizontal position then decreases with increasing inclination of plate at downward orientation. Based on the results obtained, correlations have been proposed to evaluate the Nusselt numbers of both upward and downward orientation. Validations of the present results are made through comparison with available numerical and experimental data, and a good agreement was obtained.
\end{abstract}

Keywords: Natural Convection, Laminar Flow, Vorticity and Vector Potential, Inclined Isothermal Plate

\section{Introduction}

Recently, the clarification of flow and heat transfer mechanisms of natural convection in square plates-which is often observed in many science and engineering applications such as in printed circuit board (PCB), solar cell, and thin film manufacturing chamber - is very important for both industrial applications and academic research [1].

The majority of the studies available in the literature on natural convection heat transfer from horizontal rectangular plates facing upwards have been carried out experimentally, by using air or water as working fluid [2-10]. Al-Arabi and El-Riedy [2] obtained local and average heat transfer data for horizontal plates of different shapes on the basis of the amount of steam condensate collected from compartments underneath the plates. Goldstein and Lau [3] performed mass transfer experiments with square naphthalene plates in air. Further heat transfer results for rectangular surfaces were then found by Lewandowski and Kubski [4]. Kitamura and Kimura [5] conducted an experimental study of natural convection in water from slender rectangular plates heated with uniform heat flux and equipped with fences at both longer sides to inhibit side flows, thus obtaining a two-dimensional flow field over the plates. Fluid flows and heated surface temperatures were visualized by dye tracers and liquid-crystal thermometry, respectively. The results showed that, four distinct flow regions appeared, consisting of a two-dimensional laminar boundary layer region near the leading edges, a transitional region characterized by a three-dimensional flow separation and by the attachment of ambient fluid onto the heated surface downstream of the flow separation, a fully turbulent region, and a collision region near the plate centerline. Lewandowski 
et al. [6] performed heat transfer measurements and visualized the convective flow structures in water above rectangular plates by injecting a dark-blue dye tracer through small holes distributed along the perimeter of each plate. In the same year, Pretot et al. [7] performed experiments using a rectangular plate heated with uniform heat flux. It's found that, starting from the edges of the plate, the heat transfer is characterized by a thermal boundary layer that breaks away from the surface near the center of the heated surface, giving rise to a buoyant thermal plume. Rafah Aziz [8] studied experimentally natural convection heat transfer from inclined isothermal square flat surface. Heat transfer measurements from rectangular plates facing upward were carried out in air by Martorell et al. [9], and in water by Kozanoglu and Lopez [10].

Several theoretical studies have been carried out to evaluate natural convection heat transfer from isothermal plates. The first numerical solutions of the full Navier-Stokes, continuity and energy transfer equations for natural convection from horizontal upward-facing plates were carried out by Goldstein and Lau [3]. Chen et al. [11] considered the upward facing horizontal semi-infinite plate with uniform wall temperature or uniform heat flux as a special case of a larger boundary-layer investigation of natural convection from inclined plates with variable surface temperature or heat flux. Lewandowski [12] presented a quasi-analytical solution of a theoretical model, in which the boundary layers grow from each plate-edge up to transforming into a plume at the separation point. Wei et al. [13] presented a numerical solutions of the mass, momentum and energy governing equations on two-dimensional natural convection from isothermal plates heated at both sides. Martorell et al. [9], derived two and three dimensional solutions for slender plates, concluding that although the flow near the ends of the plate is typically three-dimensional, the overall flow suffers no dramatic change with respect to the two-dimensional structure. Hassan [14] carried out a numerical study on threedimensional natural convection from inclined discs and rings at constant temperature. He also presented a numerical solution of natural convection from horizontal plate at constant wall temperature [15]. A review of the heat transfer data for free convection from upward facing heated rectangular plates is conducted by Corcione [16].

However, information and data for three-dimensional, transient natural convection from inclined square surface appears to be limited. The objective of this study is to develop a numerical simulation model to investigate the three-dimensional transient laminar natural convection from isothermal square plate subject to the influence of orientation. The Grashof numbers and inclination angles of the square plate are ranged from $10^{3}$ to $5 \times 10^{4}$ and from $0^{\circ}$ to $180^{\circ}$, respectively. As far as the fluid characteristics, the Prandtl number of 0.7 was considered. The finite difference approach is used to solve the governing equations.

\section{Governing Equations of the Present Study}

The basic equations describing the flow driven by natural convection consist of mass conservation, momentum and energy. These equations are solved numerically by finite difference approach. The numerical simulation of the problem was performed using a computer program written by FORTRAN. The Boussinesq approximation was included in the buoyancy force term so that all densities are assumed to be constant in the body force except the one in gravity term which changes with temperature.

The appropriate equations for an incompressible Newtonian fluid are [17]:

$$
\begin{gathered}
\nabla \cdot \vec{V}=0 \\
\rho \frac{D \vec{V}}{D t}=\rho \vec{g}-\nabla P+\mu \nabla^{2} \vec{V} \\
c p \cdot \rho \frac{D \Theta}{D t}=K \nabla^{2} \Theta
\end{gathered}
$$

The density is given by linearized state equation as followed [17]:

$$
\rho=\rho_{\infty}\left(1-\beta\left(T-T_{\infty}\right)\right)
$$

Equation (4) is used in buoyancy term of the momentum equation, whereas $\rho_{=} \rho_{\infty}$ is used elsewhere where $\rho_{\infty}$ is the density at reference condition. Using Boussinesq approximation, the governing equations for constant fluid property can be expressed as:

$$
\begin{gathered}
\nabla \cdot \vec{V}=0 \\
\rho_{\infty} \frac{D \vec{V}}{D t}=-\rho \vec{g} \beta\left(\Theta-\Theta_{\infty}\right)-\nabla P+\mu \nabla^{2} \vec{V} \\
c p \cdot \rho_{\infty} \frac{D \Theta}{D t}=K \nabla^{2} \Theta
\end{gathered}
$$

where $(p)$ represents the perturbation of static pressure from the hydrostatic value.

\section{Mathematical Model}

\subsection{Problem definition}

The three-dimensional geometry being solved in this study is shown in Figure1. The energy from the isothermal plate facing upward or downward orientation is dissipated by convection of ambient fluid at $T_{\infty}$. The length of square plate is $H$ and the surface is inclined with an angle of $\Phi$ with the horizontal axis. 


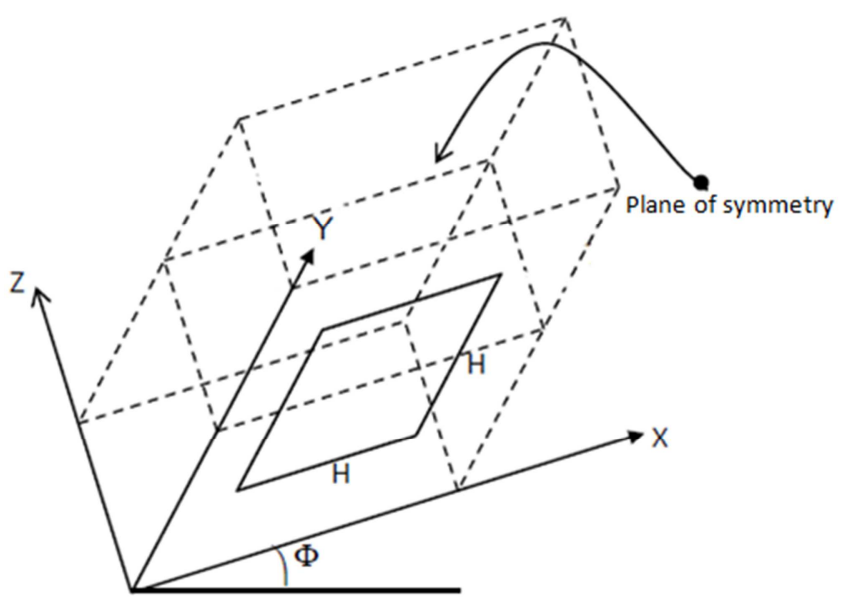

*The dashed lines represent a hypothetical outer boundary

Figure 1. Geometry of the case study

\subsection{Mathematical Model}

The continuity, momentum and energy equations for threedimensional laminar flows of an incompressible Newtonian fluid are written. Following assumptions are made: there is no viscous dissipation, the gravity acts in the vertical direction, fluid properties are constant and fluid density variations are neglected except in the buoyancy term (the Boussinesq approximation) and radiation heat exchange is negligible. The governing equations are obtained as follows:

Continuity equation

$$
\frac{\partial u}{\partial x}+\frac{\partial v}{\partial y}+\frac{\partial w}{\partial z}=0
$$

Three momentum equations

$x$-component

$$
\begin{gathered}
\frac{\partial u}{\partial t}+u \frac{\partial u}{\partial x}+v \frac{\partial u}{\partial y}+w \frac{\partial u}{\partial z}=g \beta\left(\Theta-\Theta_{\infty}\right) \sin (\Phi)-\frac{1}{\rho_{\infty}} \frac{d p}{d x} \\
+v\left(\frac{\partial^{2} u}{\partial x^{2}}+\frac{\partial^{2} u}{\partial y^{2}}+\frac{\partial^{2} u}{\partial z^{2}}\right)
\end{gathered}
$$

y-component

$$
\frac{\partial v}{\partial t}+u \frac{\partial v}{\partial x}+v \frac{\partial v}{\partial y}+w \frac{\partial v}{\partial z}=-\frac{1}{\rho_{\infty}} \frac{d p}{d y}+v\left(\frac{\partial^{2} v}{\partial x^{2}}+\frac{\partial^{2} v}{\partial y^{2}}+\frac{\partial^{2} v}{\partial z^{2}}\right)
$$

z-component

$$
\begin{aligned}
\frac{\partial w}{\partial t}+u \frac{\partial w}{\partial x}+v \frac{\partial w}{\partial y}+w \frac{\partial w}{\partial z}= & g \beta\left(\Theta-\Theta_{\infty}\right) \cos (\Phi)-\frac{1}{\rho_{\infty}} \frac{d p}{d z} \\
& +v\left(\frac{\partial^{2} w}{\partial x^{2}}+\frac{\partial^{2} w}{\partial y^{2}}+\frac{\partial^{2} w}{\partial z^{2}}\right)
\end{aligned}
$$

Energy equation

$$
\frac{\partial \Theta}{\partial t}+u \frac{\partial \Theta}{\partial x}+v \frac{\partial \Theta}{\partial y}+w \frac{\partial \Theta}{\partial z}=\alpha\left(\frac{\partial^{2} \Theta}{\partial x^{2}}+\frac{\partial^{2} \Theta}{\partial y^{2}}+\frac{\partial^{2} \Theta}{\partial z^{2}}\right)
$$

\subsection{Nondimensionalization}

Before the discretization process, we introduce the following dimensionless variables:

$$
\begin{gathered}
X=\frac{x}{H}, Y=\frac{y}{H}, Z=\frac{z}{H}, U=\frac{u H}{\alpha}, V=\frac{v H}{\alpha}, W=\frac{w H}{\alpha}, \\
\tau=\frac{t \alpha}{H^{2}}, P=\frac{p}{\rho_{\infty}(\alpha / H)^{2}}, T=\frac{\Theta-\Theta_{\infty}}{\Theta_{w}-\Theta_{\infty}}
\end{gathered}
$$

In terms of these dimensionless variables, the non-dimensional governing equations are obtained as: Continuity equation

$$
\frac{\partial U}{\partial X}+\frac{\partial V}{\partial Y}+\frac{\partial W}{\partial Z}=0
$$

Three momentum equations $X$-component

$$
\begin{aligned}
\frac{\partial U}{\partial \tau}+U \frac{\partial U}{\partial X}+V \frac{\partial U}{\partial Y}+W \frac{\partial U}{\partial Z}= & R a \sin (\Phi)-\frac{d P}{d X} \\
& +v\left(\frac{\partial^{2} U}{\partial X^{2}}+\frac{\partial^{2} U}{\partial Y^{2}}+\frac{\partial^{2} U}{\partial Z^{2}}\right)
\end{aligned}
$$

Y-component

$$
\frac{\partial V}{\partial \tau}+U \frac{\partial V}{\partial X}+V \frac{\partial V}{\partial Y}+W \frac{\partial V}{\partial Z}=-\frac{d P}{d Y}+v\left(\frac{\partial^{2} V}{\partial X^{2}}+\frac{\partial^{2} V}{\partial Y^{2}}+\frac{\partial^{2} V}{\partial Z^{2}}\right)
$$

Z-component

$$
\begin{aligned}
\frac{\partial W}{\partial \tau}+U \frac{\partial W}{\partial X}+V \frac{\partial W}{\partial Y}+W \frac{\partial W}{\partial Z}= & R a \cos (\Phi)-\frac{d P}{d Z} \\
& +v\left(\frac{\partial^{2} W}{\partial X^{2}}+\frac{\partial^{2} W}{\partial Y^{2}}+\frac{\partial^{2} W}{\partial Z^{2}}\right)
\end{aligned}
$$

Energy equation

$$
\frac{\partial T}{\partial \tau}+U \frac{\partial T}{\partial X}+V \frac{\partial T}{\partial Y}+W \frac{\partial T}{\partial Z}=\left(\frac{\partial^{2} T}{\partial X^{2}}+\frac{\partial^{2} T}{\partial Y^{2}}+\frac{\partial^{2} T}{\partial Z^{2}}\right)
$$

\subsection{Vorticity Transport Equation}

The governing equations for constant-property, incompressible flow may be cross differentiated and subtracted to eliminate pressure term, this introducing the three components of vorticity. The flowing dimensionless vorticity equations are obtained as:

$$
\begin{aligned}
& \frac{1}{\operatorname{Pr}}\left[\frac{\partial \Omega_{1}}{\partial \tau}\right]+(\vec{V} . \nabla) \Omega_{1}-\left(\Omega_{1} \cdot \nabla\right) u=\nabla^{2} \Omega_{1}+R a \cos \Phi \frac{\partial T}{\partial Y} \\
& \frac{1}{\operatorname{Pr}}\left[\frac{\partial \Omega_{2}}{\partial \tau}\right]+(\vec{V} . \nabla) \Omega_{2}-\left(\Omega_{2} . \nabla\right) v=\nabla^{2} \Omega_{2}+R a \sin \Phi \frac{\partial T}{\partial Z} \\
& -R a \cos \Phi \frac{\partial T}{\partial X}
\end{aligned}
$$

$$
\frac{1}{\operatorname{Pr}}\left[\frac{\partial \Omega_{3}}{\partial \tau}\right]+(\vec{V} . \nabla) \Omega_{3}-\left(\Omega_{3} . \nabla\right) w=\nabla^{2} \Omega_{3}-R a \sin \Phi \frac{\partial T}{\partial Y}
$$


The components of dimensionless vorticity used here are defined as the curl of the dimensionless velocity in term of dimensionless coordinates

$$
\begin{gathered}
\vec{\Omega}=\nabla \times \vec{V} \\
\Omega_{1}=\frac{\partial W}{\partial Y}-\frac{\partial V}{\partial Z} \\
\Omega_{2}=\frac{\partial U}{\partial Z}-\frac{\partial W}{\partial X} \\
\Omega_{3}=\frac{\partial V}{\partial X}-\frac{\partial U}{\partial Y}
\end{gathered}
$$

To calculate the velocity from the vorticity, it is convenient to introduce a vector potential $\vec{\Psi}$, which may be looked upon as the three dimensional counterpart of two dimension stream function. The components of the velocity are related to the components of dimensionless vector potential. The dimensionless vector potential is defined such that its curl equals the dimensionless velocity vector:

$$
\begin{gathered}
\vec{V}=\nabla \times \vec{\Psi} \\
U=\frac{\partial \Psi_{3}}{\partial Y}-\frac{\partial \Psi_{2}}{\partial Z} \\
V=\frac{\partial \Psi_{1}}{\partial Z}-\frac{\partial \Psi_{3}}{\partial X} \\
W=\frac{\partial \Psi_{2}}{\partial X}-\frac{\partial \Psi_{1}}{\partial Y}
\end{gathered}
$$

The vector potential that satisfies the continuity equation is written as:

$$
\nabla \cdot \vec{V}=\nabla \cdot(\nabla \times \vec{\Psi})
$$

and it must be solenoid, i.e.

$$
\frac{\partial^{2} \Psi_{1}}{\partial X^{2}}+\frac{\partial^{2} \Psi_{1}}{\partial Y^{2}}+\frac{\partial^{2} \Psi_{1}}{\partial Z^{2}}=0
$$

The vorticity is then related to the vector potential as follows:

$$
\begin{aligned}
& -\Omega_{1}=\frac{\partial^{2} \Psi_{1}}{\partial X^{2}}+\frac{\partial^{2} \Psi_{1}}{\partial Y^{2}}+\frac{\partial^{2} \Psi_{1}}{\partial Z^{2}} \\
& -\Omega_{2}=\frac{\partial^{2} \Psi_{2}}{\partial X^{2}}+\frac{\partial^{2} \Psi_{2}}{\partial Y^{2}}+\frac{\partial^{2} \Psi_{2}}{\partial Z^{2}} \\
& -\Omega_{3}=\frac{\partial^{2} \Psi_{3}}{\partial X^{2}}+\frac{\partial^{2} \Psi_{3}}{\partial Y^{2}}+\frac{\partial^{2} \Psi_{3}}{\partial Z^{2}}
\end{aligned}
$$

\subsection{Boundary Conditions}

The boundary conditions of the system shown in Figure1 is detailed as:

Temperature

At $Z=0 \quad 0 \leq X \leq 1 \quad$ and $\quad 0 \leq Y \leq 0.5 \quad, T=1$

At $Z=2 \quad 0 \leq X \leq 1$ and $0 \leq Y \leq 0.5 \quad, T=0$

At $X=0$ and $X=1 \quad 0 \leq Z \leq 2$ and $0 \leq Y \leq 0.5, T=0$

At $Y=0$ and $Y=0.5 \quad 0 \leq Z \leq 2$ and $0 \leq X \leq 1, T=0$

Vector potential

At $Z=0 \quad 0 \leq X \leq 1 \quad$ and $\quad 0 \leq Y \leq 0.5$

$\Psi_{1}=\Psi_{2}=\frac{\partial \Psi_{3}}{\partial Z}=0$

At $Z=2 \quad 0 \leq X \leq 1 \quad$ and $\quad 0 \leq Y \leq 0.5$

$\Psi_{1}=\Psi_{2}=\Psi_{3}=0$

At $X=0$ and $X=1 \quad 0 \leq Z \leq 2$ and $0 \leq Y \leq 0.5$

$\Psi_{1}=\Psi_{2}=\Psi_{3}=0$

At $Y=0$ and $Y=0.5 \quad 0 \leq Z \leq 2$ and $\quad 0 \leq X \leq 1$

$\Psi_{1}=\Psi_{2}=\Psi_{3}=0$

Vorticity Vector

At $Z=0 \quad 0 \leq X \leq 1 \quad$ and $\quad 0 \leq Y \leq 0.5$

$\Omega_{1}=-\frac{\partial V}{\partial Z}, \Omega_{2}=\frac{\partial U}{\partial Z}, \quad \Omega_{3}=0$

At $Z=2 \quad 0 \leq X \leq 1 \quad$ and $\quad 0 \leq Y \leq 0.5$

$\Omega_{1}=\Omega_{2}=\Omega_{3}=0$

At $X=0$ and $\quad X=1 \quad 0 \leq Z \leq 2$ and $0 \leq Y \leq 0.5$

$\Omega_{1}=\Omega_{2}=\Omega_{3}=0$

At $Y=0$ and $Y=0.5 \quad 0 \leq Z \leq 2$ and $\quad 0 \leq X \leq 1$

$\Omega_{1}=\Omega_{2}=\Omega_{3}=0$

\section{Numerical Solution}

The numerical simulation of three-dimensional transient natural convection flow from inclined isothermal plate is done using (ADI) method for vorticity and temperature equations, and by (SOR) method for the vector potential equations.

The energy and vorticity equations (17-20) can be written as follow:

$$
\frac{\partial T}{\partial \tau}=\frac{\partial^{2} T}{\partial X^{2}}-U \frac{\partial T}{\partial X}+\frac{\partial^{2} T}{\partial Y^{2}}-V \frac{\partial T}{\partial Y}+\frac{\partial^{2} T}{\partial Z^{2}}-W \frac{\partial T}{\partial Z}
$$




$$
\begin{gathered}
\frac{\partial \Omega_{1}}{\partial \tau}=p r \frac{\partial^{2} \Omega_{1}}{\partial X^{2}}-U \frac{\partial \Omega_{1}}{\partial X}+p r \frac{\partial^{2} \Omega_{1}}{\partial Y^{2}}-V \frac{\partial \Omega_{1}}{\partial Y} \\
+p r \frac{\partial^{2} \Omega_{1}}{\partial Z^{2}}-W \frac{\partial \Omega_{1}}{\partial Z}+S_{1} \\
\frac{\partial \Omega_{2}}{\partial \tau}=p r \frac{\partial^{2} \Omega_{2}}{\partial X^{2}}-U \frac{\partial \Omega_{2}}{\partial X}+p r \frac{\partial^{2} \Omega_{2}}{\partial Y^{2}}-V \frac{\partial \Omega_{2}}{\partial Y} \\
+p r \frac{\partial^{2} \Omega_{2}}{\partial Z^{2}}-W \frac{\partial \Omega_{2}}{\partial Z}+S_{2} \\
\frac{\partial \Omega_{3}}{\partial \tau}=p r \frac{\partial^{2} \Omega_{3}}{\partial X^{2}}-U \frac{\partial \Omega_{3}}{\partial X}+p r \frac{\partial^{2} \Omega_{3}}{\partial Y^{2}}-V \frac{\partial \Omega_{3}}{\partial Y} \\
+p r \frac{\partial^{2} \Omega_{3}}{\partial Z^{2}}-W \frac{\partial \Omega_{3}}{\partial Z}+S_{3}
\end{gathered}
$$

where

$$
\begin{gathered}
S_{1}=R a \cos \Phi \frac{\partial T}{\partial Y} \\
S_{2}=R a \sin \Phi \frac{\partial T}{\partial Z}-R a \cos \Phi \frac{\partial T}{\partial X} \\
S_{3}=-R a \sin \Phi \frac{\partial T}{\partial Y}
\end{gathered}
$$

The three equations of vector potential components $\Psi_{1}, \Psi_{2}$ and $\Psi_{3}$ can be solved at any time-step using a point iterative successive over relaxation method [18].

$$
\begin{gathered}
\Psi_{(i, j, k)}=\frac{1}{b}\left(\Psi_{(i-1, j, k)}+\Psi_{(i+1, j, k)}+\Psi_{(i, j-1, k)}+\Psi_{(i, j+, k)}\right. \\
\left.+\Psi_{(i, j, k-1)}+\Psi_{(i, j, k+1)}+S\right)
\end{gathered}
$$

A relaxation factor $W_{\Psi}$ was defined as follows,

$$
\Psi_{(i, j, k)}^{n(s+1)}=\Psi_{(i, j, k)}^{n}+W_{\Psi}\left(\Psi_{(i, j, k)}^{n}-\Psi_{(i, j, k)}^{n(s)}\right)
$$

Where the counter (s) refers to the number of successive point iterations performed at the nth time step, and $\Psi_{(i, j, k)}^{n(s+1)}$ is the value of the component $\Psi$ at the nth time step after (s+1) iterations. The value of $\Psi_{(i, j, k)}^{n(s+1)}$ are resubstituted in to equation (35) where is then solved with equation (36) until the following convergence criterion is satisfied:

$$
\sum\left(\Psi_{(i, j, k)}^{n(s+1)}-\Psi_{(i, j, k)}^{n(s)}\right) \leq \varepsilon
$$

where $\varepsilon=10^{-3}$, the relaxation factor $W_{\Psi}$ is 1.7 for trial and error for the present work.

The local Nusselt number is defined as

$$
N u=-\left.\frac{\partial T}{\partial Z}\right|_{Z=0}
$$

Equation (38) is solved using forward finite difference for four points as follow:

$$
N u=-\frac{1}{6 \Delta Z}\left(11 T_{(i, j, 1)}-18 T_{(i, j, 2)}+9 T_{(i, j, 3)}-2 T_{(i, j, 1)}\right)
$$

Average Nusselt number is defined as

$$
\overline{N u}=\frac{1}{A} \iint_{A} N u d A
$$

This integration can be carried out using trapezoidal rule [18].

\section{Result and Discussion}

In the present study, a finite difference method is applied to find the numerical solution of three-dimensional transient natural convection from isothermal square plate subject to the influence of orientation.

Temperature fields, local and average Nusselt Number distribution over the heated surface are examined for Grashof numbers and inclination angles ranged from $10^{3}$ to $5 \times 10^{4}$ and from $0^{\circ}$ to $180^{\circ}$, respectively. As far as the ambient fluid characteristics, $P r=0.7$ was considered.

\subsection{Temperature Fields}

Figures 2 and 3 show that the isothermal lines of the heated surface at two plans $(z-x)$ and $(z-y)$ at $x=1.0$ and Grashof numbers ranged from $10^{4}$ to $5 \times 10^{4}$. The temperature fields are assumed to have a symmetric nature with respect to vertical plane. It's clear that the temperature gradient above the heated surface of square plate increases rapidly in the vicinity of free edge. The isotherms show formation of a boundary layer heat transfer along the heated surface. The temperature gradients are concentrated just above the center of the heated surface, where a thermal plume is formed. 

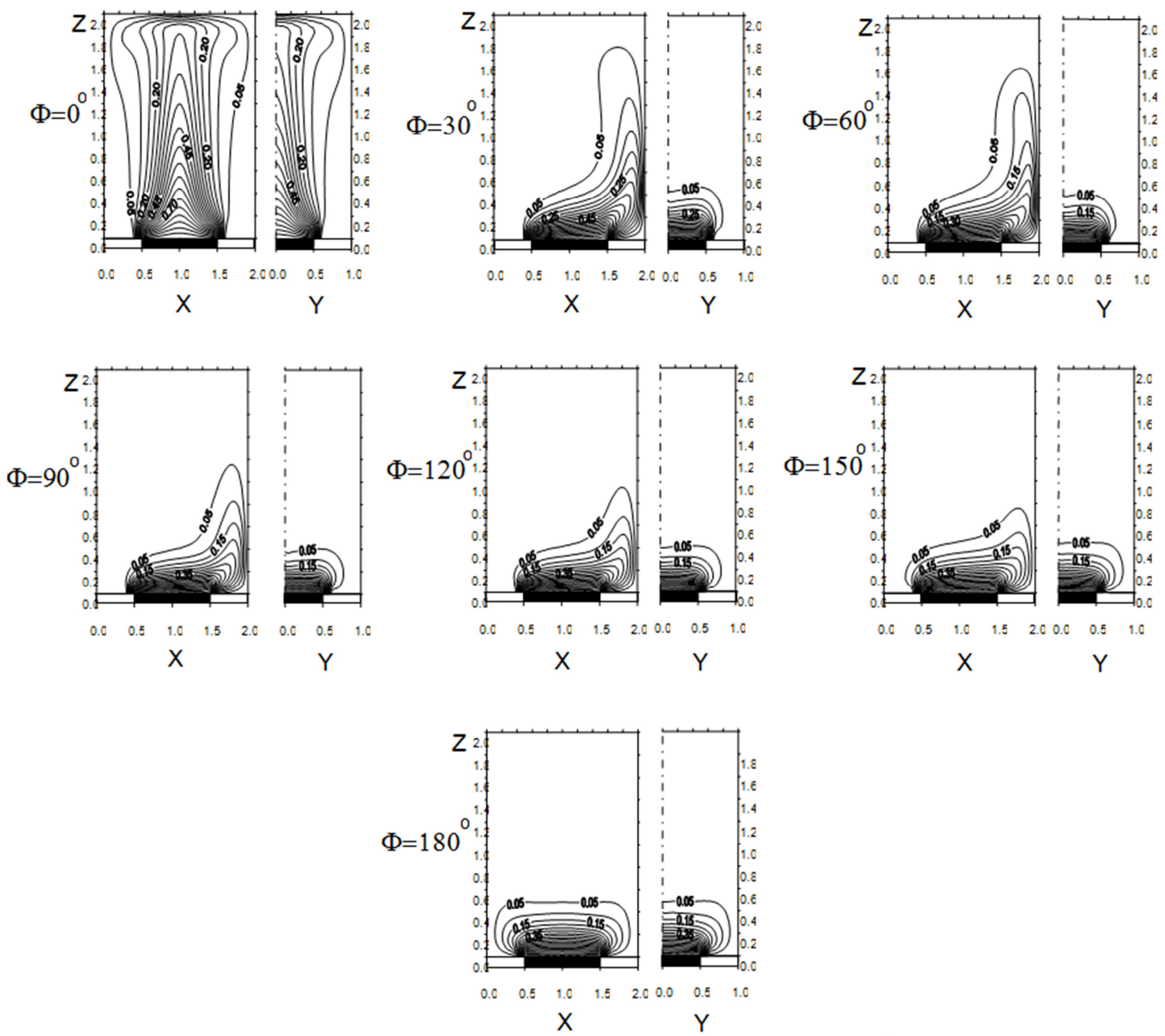

Figure 2. Isotherms plots of square plate at different inclination angles $\left(\mathrm{Gr}=10^{4}\right)$

As inclination from horizontal increases, the bouncy component along the surface arises and causes an increase in the velocity and also a plume shifted completely to the upper part of the plate. The density of fluid decreases in the region between the center and upper edge, while the velocity of the fluid motion continuous to increase. All that leads to ascending flow after the center, therefore the boundary layer thickness continues to increase until the upper edge. However, when square plate is heated at both horizontal facing upward and downward orientation it has been found that a plume rising from the heated surface and there is significant change on temperature field when the orientation is changed. The small effect of convection mode is existed at low Grashof number. Further increase of Grashof number, the isothermal lines becomes distorted, resulting in an increase in heat transfer rates. It's clear from Figure3 that the effect of convection for Grashof number of $5 \times 10^{4}$ is obvious by appearance of plume over the heated surface and the effect of inclination angle on the shape of isothermal lines over the plate. 

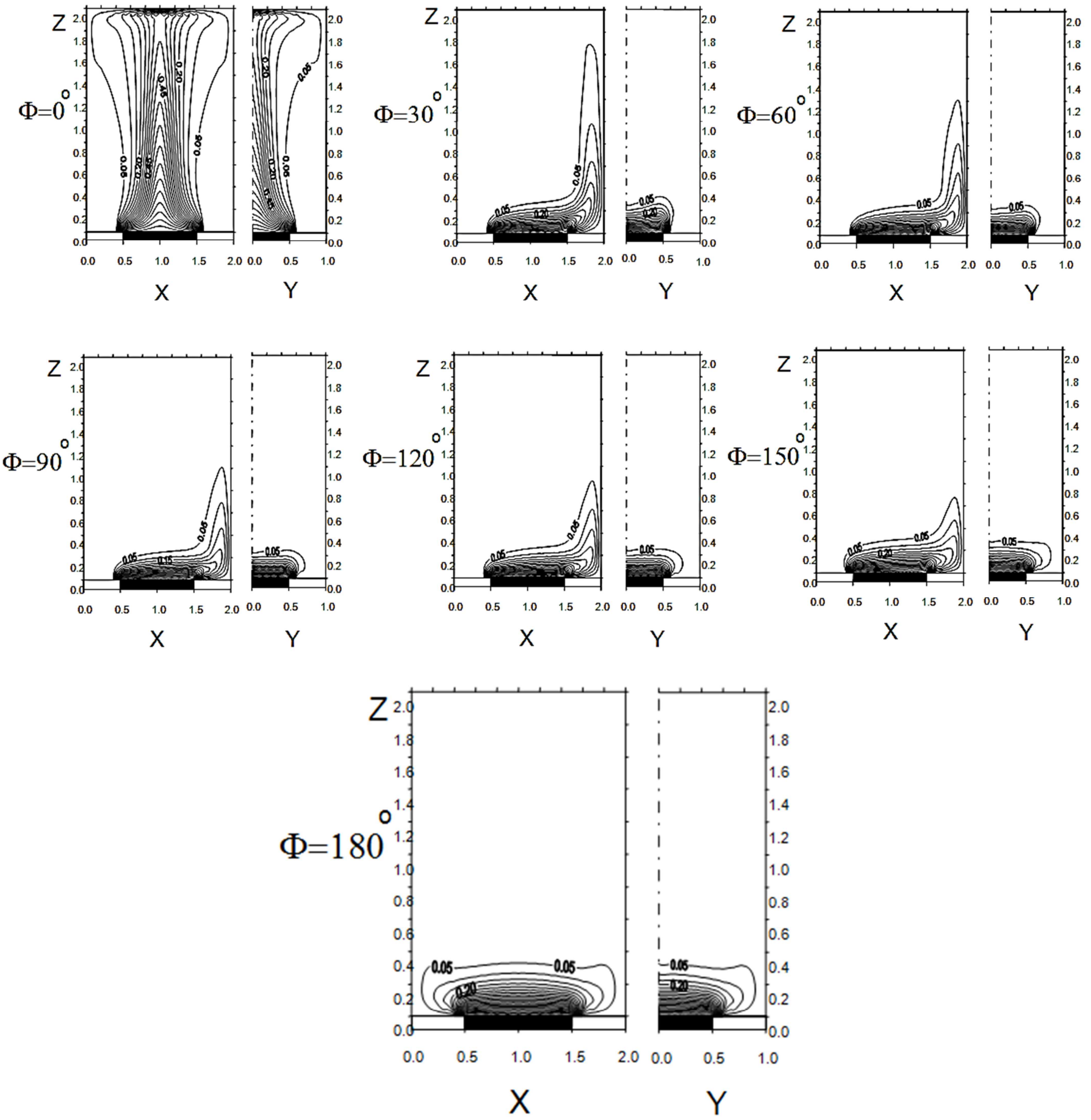

Figure 3. Isotherms plots of square plate at different inclination angles $\left(\mathrm{Gr}=5 \times 10^{4}\right)$

\subsection{Local Nusselt Number}

The distribution of local Nusselt number for Grashof number of $10^{3}$ to $5 \times 10^{4}$ is shown in Figure 4 at facing upward and downward orientation. At $G r=10^{3}$, it's found that, the local Nusselt number has very small values because of small effect of convection mode of hear transfer exists, since low Grashof number the mode of heat transfer is conduction only. At $G r=10^{4}$ and $G r=5 \times 10^{4}$, the increased contribution of convection is observed and local Nusselt number values increase at all.

The higher values of local Nusselt number occur at leading edge of the square plate and reach to maximum value at vertical position. Depending on the heated surface orientation, the heat transfer rate of these two orientations shows a competitive nature. It is observed that, there is a gradual increase in the local values of Nusselt numbers compared to the horizontal position and the horizontal upward and downward orientations yield the lowest heat transfer coefficients. 

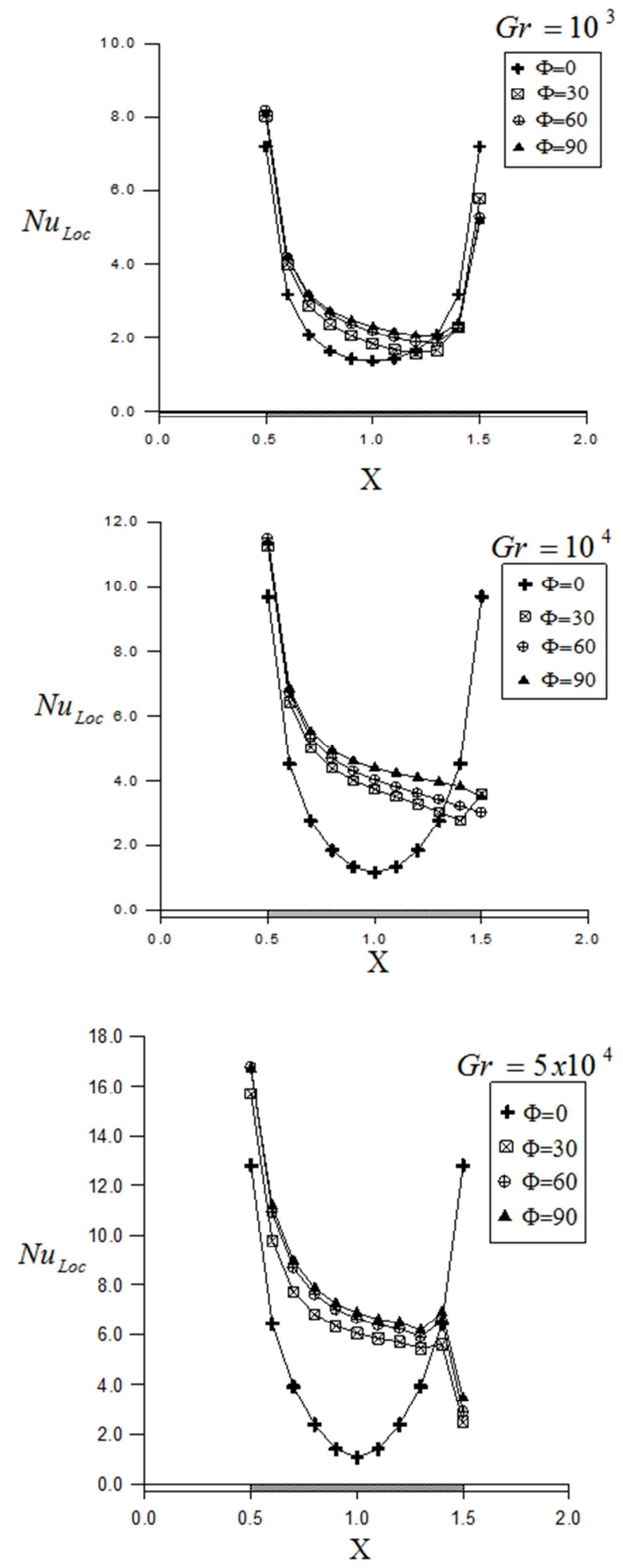

(a) Upward Heating
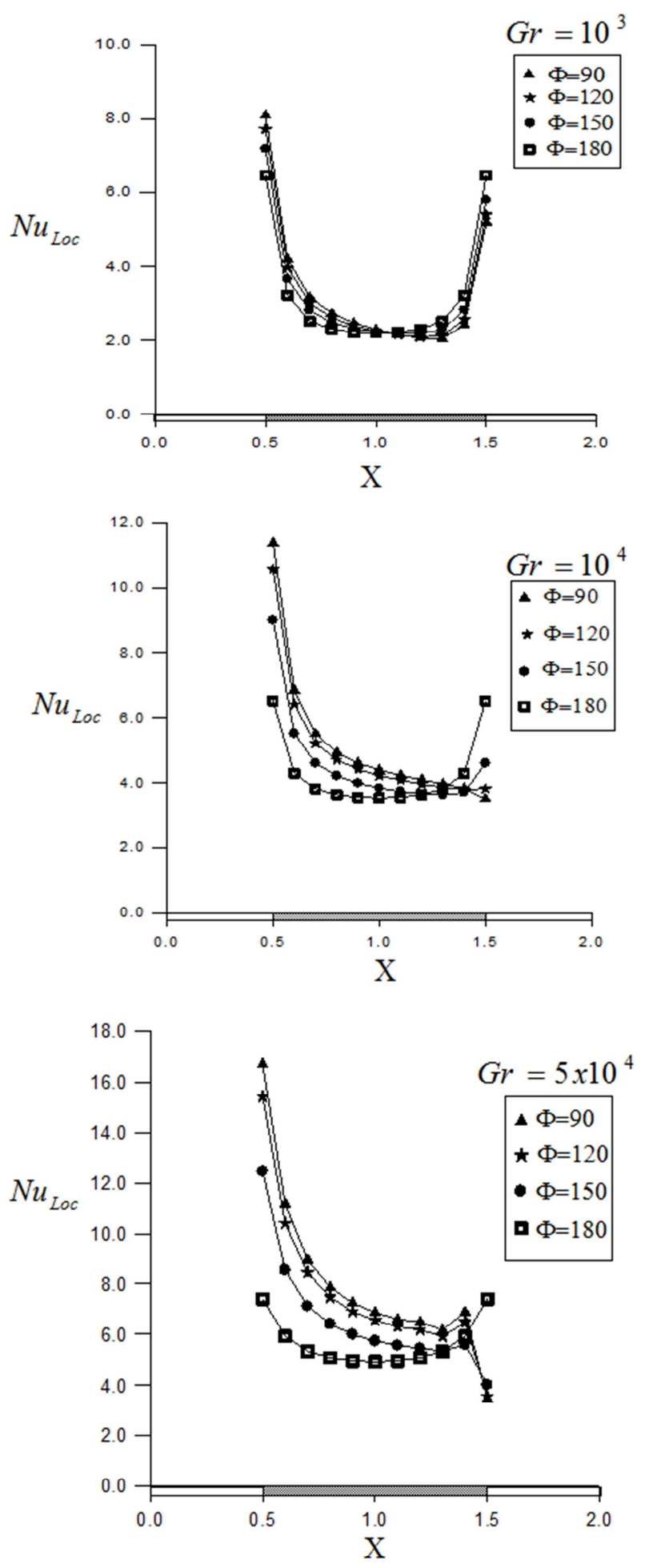

(b) Downward Heating

Figure 4. Local Nusselt number in the line of symmetry $\left(\mathrm{Gr}=10^{3}, 10^{4}, 5 \times 10^{4}\right)$

\subsection{Average Nusselt Number}

Based on the results obtained, correlations have been proposed to evaluate the Nusselt numbers for both upward and downward orientation as shown in Figure (5). The form of the proposed correlation takes into account the variation of the inclination angle as shown in Figure (6). It's found that, the average Nusselt number shows fairly large dependence on the 
inclination angles of heated square plate at different values of Rayleigh number. It is also found that, the Nusselt number values show strong dependence on inclination angle of the square plate and it reaches to the maximum value at the
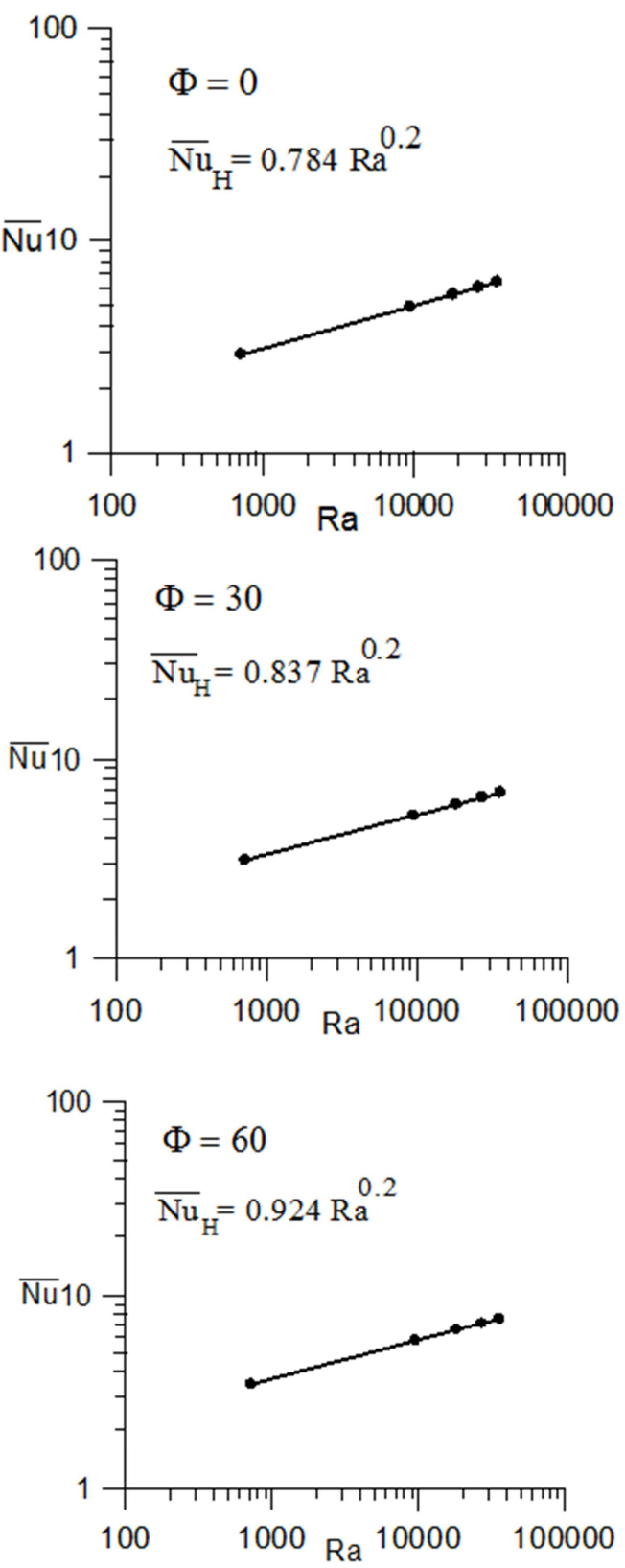

vertical position. The average Nusselt number increases by $20 \%$ at the vertical position then decrease with increase of inclination of plate at downward orientation.
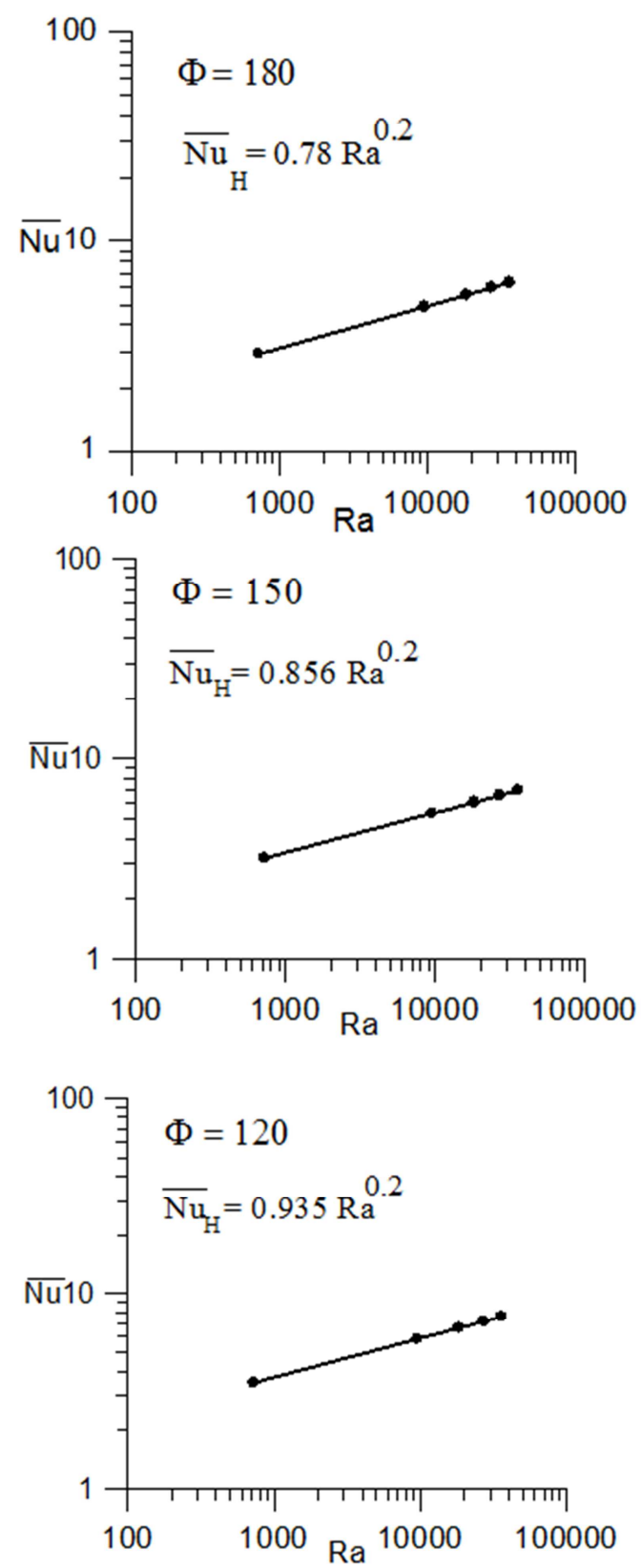

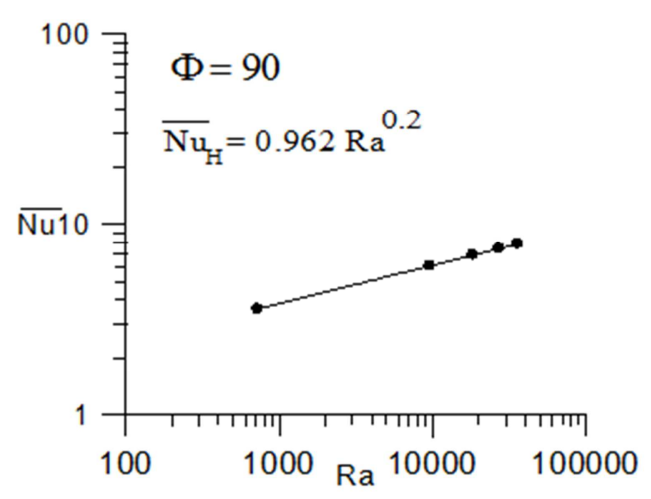

Figure 5. Nusselt number vs. Rayleigh number correlations at different inclination angles 


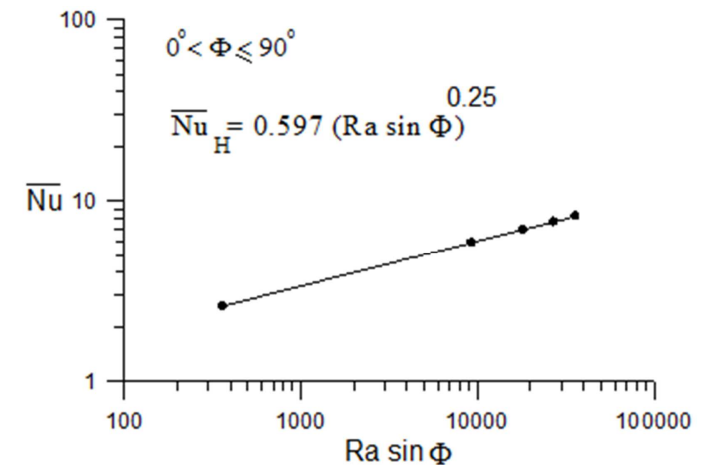

(a) Upward heating $0^{\circ} \leq \Phi \leq 90^{\circ}$

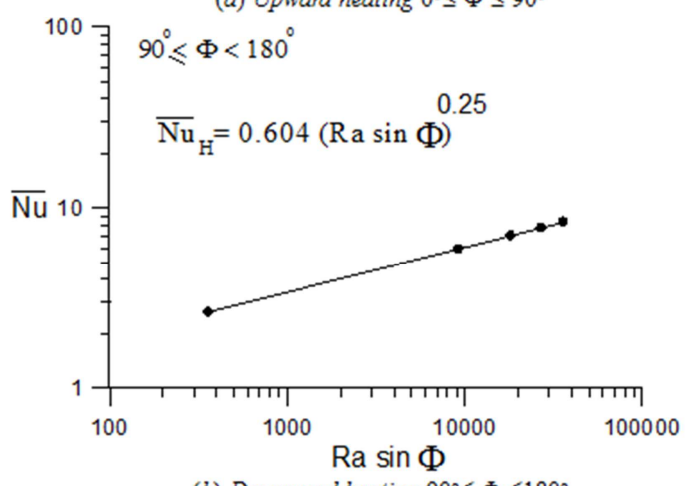

(b) Downward heating $90^{\circ} \leq \Phi \leq 180^{\circ}$

Figure 6. Nusselt number vs. Rayleigh number correlations

\subsection{Comparison with Available Previous Work}

The validation of the obtained results is made through comparison with available numerical and experimental data. Figure 7 shows a comparison between the present study with available previous literature $[3,8]$ in case of horizontal position $\left(\Phi=0^{\circ}\right)$ facing upward. It can be seen that, a good agreement is achieved between the present results and the available experimental results. Figure 8 shows a convergence between the present numerical result with literature experimental data [3] at horizontal position $\left(\Phi=180^{\circ}\right)$ facing downward. In case of vertical position $\left(\Phi=90^{\circ}\right)$, it's found that there is excellence agreement was obtained with the available numerical data [14], see Figure 9.

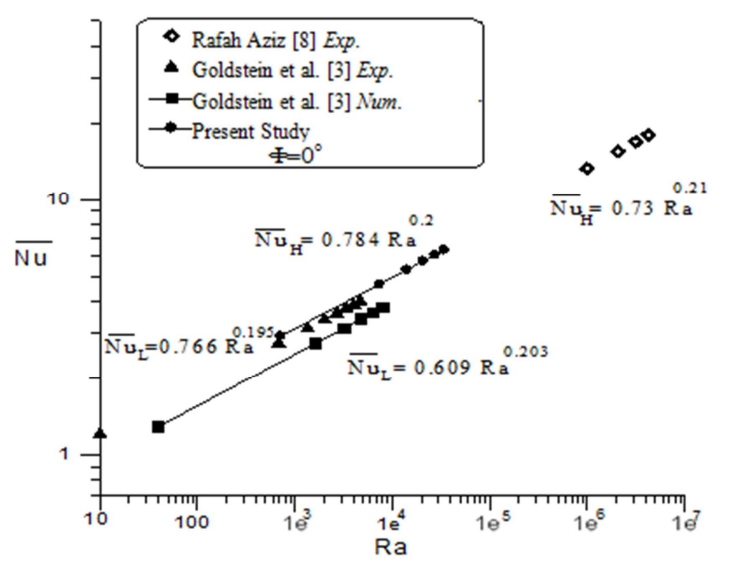

Figure 7. Comparison of average Nusselt number with available theoretical and experimental data $\left(\Phi=0^{\circ}\right)$

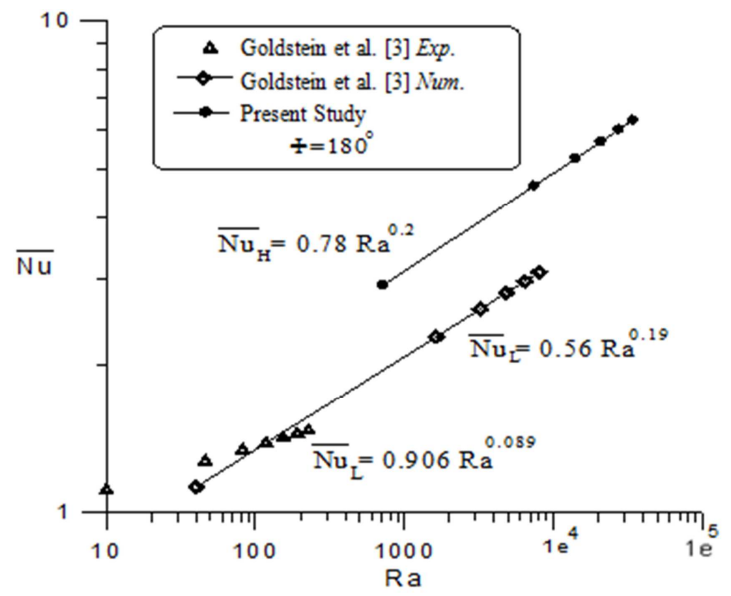

Figure 8. Comparison of average Nusselt number with available theoretical and experimental data $\left(\left(\Phi=180^{\circ}\right)\right.$

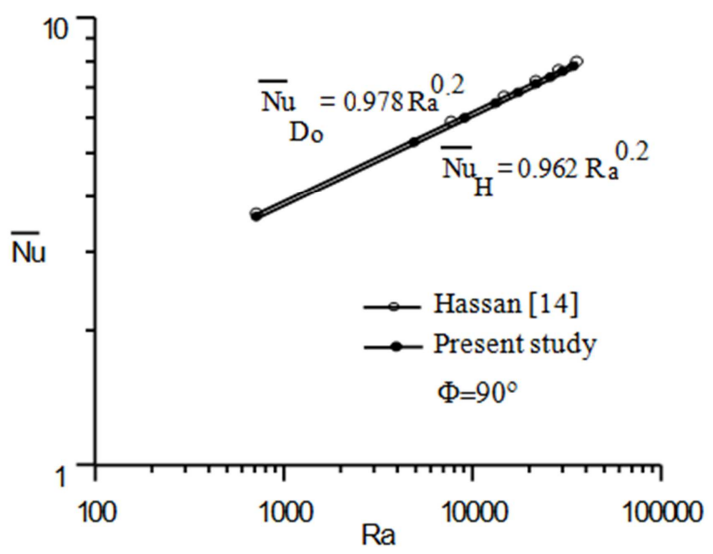

Figure 9. Comparison of average Nusselt number with available theoretical data over vertical square plate

\section{Conclusion}

There is significant effect on temperature filed and Nusselt number when the orientation of the heated surface is changed. It's also observed that, the Nusselt number shows a strong function of inclination angle. There is a difference in heat transfer rates between the upward and downward orientation where the maximum values are obtained at the vertical position. Finally, the relation between average Nusselt number and Rayleigh number are correlated and takes into account the inclination of the heated surface. Finally, the horizontal upward and downward orientations yield the lowest heat transfer rates.

\section{Nomenclature}

A Area of the heated surface $\left(\mathrm{m}^{2}\right)$

g Gravitational vector $\left(\mathrm{m}^{2} / \mathrm{s}\right)$

$\mathrm{h} \quad$ Heat transfer coefficient $\left(\mathrm{W} / \mathrm{m}^{2} . \mathrm{K}\right)$

$\mathrm{H}$ Length of the square plate $(\mathrm{m})$

$\mathrm{k}$ Thermal conductivity $(\mathrm{W} / \mathrm{m} . \mathrm{K})$

$\mathrm{T}$ Dimensionless temperature $=\left(\Theta-\Theta_{\infty}\right) /\left(\Theta_{\mathrm{w}}-\Theta_{\infty}\right)$

$\mathrm{U}$ Dimensionless X-component of velocity $=\mathrm{uH} / \alpha$ 
$\mathrm{V}$ Dimensionless Y-component of velocity $=\mathrm{vH} / \alpha$

W Dimensionless Z-component of velocity $=\mathrm{wH} / \alpha$

$\mathrm{X}$ Dimensionless X-coordinate $=\mathrm{x} / \mathrm{H}$

$\mathrm{Y}$ Dimensionless Y-coordinate $=\mathrm{y} / \mathrm{H}$

$\mathrm{Z} \quad$ Dimensionless Z-coordinate $=\mathrm{z} / \mathrm{H}$

\section{Greek Symbols}

$\alpha$ Thermal diffusivity of air $\left(\mathrm{m} / \mathrm{s}^{2}\right)$

$\beta$ Coefficient of thermal expansion $\left(\mathrm{K}^{-1}\right)$

$v$ Kinematics viscosity of air $\left(\mathrm{m} / \mathrm{s}^{2}\right)$

$\tau \quad$ Dimensionless time $=\mathrm{t} \alpha / \mathrm{H}^{2}$

$\vec{\Psi} \quad$ Dimensionless vector potential

$\Omega \quad$ Dimensionless vorticity vector

$\Theta \quad$ Temperature $(\mathrm{K})$

$\Phi \quad$ Angle of inclination

\section{Subscripts \\ $\infty \quad$ Ambient \\ w Value of surface \\ - Average value \\ 1 Vector component in X-direction \\ 2 Vector component in Y-direction \\ 3 Vector component in Z-direction}

\section{Dimensionless Numbers}

Gr Grashof number $=\mathrm{g} \beta\left(\Theta_{\mathrm{w}}-\Theta_{\infty}\right) \mathrm{H}^{3} / \mathrm{v}^{2}$

Ra Rayleigh number $=\mathrm{Gr} \cdot \operatorname{Pr}=\mathrm{g} \beta\left(\Theta_{\mathrm{w}}-\Theta_{\infty}\right) \mathrm{H}^{3} / \mathrm{v} \alpha$

$\mathrm{Nu}$ Local Nusselt number $=\mathrm{hH} / \mathrm{k}$

Pr Prandtl number $=v / \alpha$

\section{References}

[1] Fu, Wu-Shung, Wei-Hsiang Wang, and Chung-Gang Li, An investigation of natural convection in parallel square plates with a heated top surface by a hybrid boundary condition, International Journal of Thermal Sciences 84 (2014) $48-61$.

[2] M. Al-Arabi, M. K. El-Riedy, Natural convection heat transfer from isothermal horizontal plates of different shapes, Int. J. Heat Mass Transfer 19 (1976):1399-1404.

[3] Goldstein, R. J., and Kei-Shun Lau, Laminar natural convection from a horizontal plate and the influence of plate-edge extensions, Journal of Fluid Mechanics 129 (1983): 55-75.

[4] Lewandowski, W. M., and P. Kubski, Effect of the use of the balance and gradient methods as a result of experimental investigations of natural convection action with regard to the conception and construction of measuring apparatus, Heat and Mass Transfer 18 (1984): 247-256.
[5] Kitamura, Kenzo, and Fumiyoshi Kimura, Heat transfer and fluid flow of natural convection adjacent to upward-facing horizontal plates, International journal of heat and mass transfer 38 (1995): 3149-3159.

[6] Lewandowski, Witold M., et al., Free convection heat transfer and fluid flow above horizontal rectangular plates, Applied energy 66 (2000): 177-197.

[7] Pretot, S., B. Zeghmati, and G. Le Palec, Theoretical and experimental study of natural convection on a horizontal plate, Applied thermal engineering 20 (2000): 873-891.

[8] Rafah Aziz, Instruction system to study free convection heat transfer from isothermal square flat surface, 2000, M.Sc. Thesis, University of Technology, Baghdad.

[9] Martorell, Ingrid, Joan Herrero, and Francesc X. Grau, Natural convection from narrow horizontal plates at moderate Rayleigh numbers, International Journal of heat and mass transfer 46 (2003): 2389-2402.

[10] Kozanoglu, Bulent, and Jorge Lopez, Thermal boundary layer and the characteristic length on natural convection over a horizontal plate, Heat and mass transfer 43 (2007): 333-339.

[11] Chen, T. S., Hwa-Chong Tien, and Bassem F. Armaly. "Natural convection on horizontal, inclined, and vertical plates with variable surface temperature or heat flux." International journal of heat and mass transfer 29 (1986): 1465-1478.

[12] Lewandowski, Witold M. "Natural convection heat transfer from plates of finite dimensions." International journal of heat and mass transfer 34 (1991): 875-885.

[13] Wei, J. J., B. Yu, and Y. Kawaguchi, Simultaneous natural-convection heat transfer above and below an isothermal horizontal thin plate, Numerical Heat Transfer 44 (2003): $39-58$.

[14] Ayad K. Hassan, Prediction of three dimensional natural convection from heated disk and rings at constant temperature, Engineering and Technology 5 (2003):229-248.

[15] Ayad K. Hassan, Prediction of three dimensional natural convection from a horizontal isothermal square plate, Journal of Engineering 13 (2006):742-755.

[16] Corcione Massimo, Heat transfer correlations for free convection from upward-facing horizontal rectangular surfaces, WSEAS Transactions on Heat and Mass Transfer 2 (2007): 48-60.

[17] Torrance K.E, Numerical Methods in Heat Transfer, Handbook of Heat Transfer Fundamentals, McGraw-Hill, 2nd Edition, 1985 .

[18] Gerald, Curtis F., Patrick O. Wheatley, and Fengshan Bai. Applied numerical analysis. New York: Addison-Wesley, 1989. 